\title{
Direct inhibition of the sodium/hydrogen exchanger after prolonged regional ischemia improves contractility on reperfusion independent of myocardial viability
}

\author{
William M. Yarbrough, MD \\ Rupak Mukherjee, PhD \\ G. Patricia Escobar, DVM \\ Joseph T. Mingoia, BS \\ Jeffrey A. Sample, BS \\ Jennifer W. Hendrick, BS \\ Kathryn B. Dowdy, BS \\ Julie E. McLean, BS \\ Robert E. Stroud, BS \\ Francis G. Spinale, MD, PhD
}

From the Division of Cardiothoracic Surgery, Medical University of South Carolina, Charleston, SC.

This study was supported by the National Heart, Lung, and Blood Institute grants HL45024, HL-97012, and PO1-48788; the National Institute of Health Postdoctoral Training grant HL-07260; and a basic research grant provided by Mitsubishi Pharma Corporation.

Received for publication Feb 5, 2003; revisions requested March 24, 2003; revisions received March 31, 2003; accepted for publication May 19, 2003.

Address for reprints: Francis G. Spinale, $\mathrm{MD}, \mathrm{PhD}$, Cardiothoracic Research, Strom Thurmond Research Building, 770 MUSC Complex, Suite 625, Medical University of South Carolina, Charleston, SC 29425.

J Thorac Cardiovasc Surg 2003;126: 1489-97

Copyright $\odot 2003$ by The American Association for Thoracic Surgery

$0022-5223 / 2003 \$ 30.00+0$

doi:10.1016/S0022-5223(03)00811-0
Background: A mechanism for myocardial dysfunction after ischemia and reperfusion is $\mathrm{Na}^{+} / \mathrm{H}^{+}$exchanger activation. Although past in vivo models of limited ischemia and reperfusion intervals demonstrate that $\mathrm{Na}^{+} / \mathrm{H}^{+}$exchanger inhibition confers myocardial protection when administered at the onset of ischemia, the effect of $\mathrm{Na}^{+} / \mathrm{H}^{+}$exchanger inhibition on myocardial function after prolonged ischemia and reperfusion remains unknown. This investigation tested the hypothesis that $\mathrm{Na}^{+} / \mathrm{H}^{+}$exchanger inhibition instituted at reperfusion and after prolonged coronary occlusion in pigs would influence myocardial contractility independent of myocardial viability.

Methods: A coronary snare and sonomicrometry crystals were placed in pigs $(\mathrm{n}=$ $21,32 \mathrm{~kg}$ ). Coronary occlusion was instituted for 120 minutes followed by reperfusion for 180 minutes. At 105 minutes of ischemia, pigs were randomized to ischemia and reperfusion only (saline solution, $\mathrm{n}=11$ ) or $\mathrm{Na}^{+} / \mathrm{H}^{+}$exchanger inhibition (HOE-642, $3 \mathrm{mg} / \mathrm{kg}$ intravenously, $\mathrm{n}=10$ ). Myocardial injury was determined by tissue staining and measurement of plasma myocyte-specific enzymes. Myocardial contractility was determined by calculation of the regional end-systolic pressure-dimension relation (millimeters of mercury per centimeter) and by assessment of interregional shortening.

Results: Infarct size was not different between groups $(39 \% \pm 6 \%, P=.26)$. Moreover, at 180 minutes of reperfusion, plasma troponin-I and creatine kinase MB values had increased to identical levels in the ischemia and reperfusion-only and $\mathrm{Na}^{+} / \mathrm{H}^{+}$exchanger inhibition groups (300 \pm 35 and $50 \pm 6 \mathrm{ng} / \mathrm{mL}$, respectively). At 90 minutes of ischemia, regional end-systolic pressure-dimension relation decreased from baseline $(5.7 \pm 0.5$ versus $2.7 \pm 0.3, P<.05)$ in the area at risk. By 30 minutes of reperfusion, regional end-systolic pressure-dimension relation decreased further in the ischemia and reperfusion-only group $(1.6 \pm 0.2, P<.05)$, but improved with $\mathrm{Na}^{+} / \mathrm{H}^{+}$exchanger inhibition $(4.4 \pm 0.7, P<.05)$.

Conclusions: $\mathrm{Na}^{+} / \mathrm{H}^{+}$exchanger inhibition instituted at reperfusion improved contractility independent of myocardial viability as assessed by absolute infarct size and myocyte-specific enzyme release. Thus, modulation of $\mathrm{Na}^{+} / \mathrm{H}^{+}$exchanger activity in the setting of prolonged ischemia and reperfusion may hold therapeutic potential. 


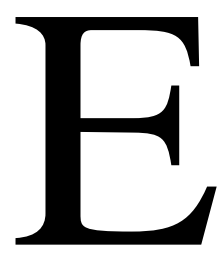

fforts to attenuate myocardial injury and improve survival after the onset of myocardial ischemia hinge on prompt restoration of coronary blood flow using both pharmacologic and mechanical interventions. ${ }^{1,2}$ However, reestablishment of myocardial perfusion is not without adverse consequences. ${ }^{3,4}$ Specifically, reperfusion of ischemic myocardium may ignite a cascade of cellular events known to result in myocyte injury through a process distinct from that encountered by ischemia alone., This process, ischemia-reperfusion (IR) injury, is likely attributable to perturbations in calcium homeostasis within myocytes and can result in contractile dysfunction. ${ }^{4,7}$ Past in vitro studies demonstrate that a contributory mechanism for IR dysfunction is increased activation of the $\mathrm{Na}^{+} / \mathrm{H}^{+}$exchanger (NHE) causing an intracellular $\mathrm{Na}^{+}$shift and subsequent calcium accumulation. ${ }^{8-10}$ Calcium overload is known to prompt cell death through several mechanisms, which include protease activation and direct mitochondrial injury. ${ }^{4,11}$ Moreover, the role of the activated NHE as a causative entity in the pathogenesis of IR injury has been supported by numerous investigations in which intact models of myocardial IR injury were used. ${ }^{12-18}$ These preclinical investigations demonstrated that NHE inhibition (NHEI) conferred myocardial protection, as evidenced by reduction in infarct size after myocardial reperfusion. ${ }^{13-18}$ However, while providing proof of concept and the impetus for the execution of several recent clinical trials, ${ }^{19-22}$ these preclinical studies are not directly translatable into clinical contexts. Specifically, the majority of these experimental studies used NHEI before the onset of limited ischemic intervals, a scenario that is infrequently realized in clinical settings. Therefore, whether and to what degree NHEI influences myocardial contractility and viability when instituted at reperfusion after prolonged periods of regional ischemia remains unexplored. Accordingly, this study used a porcine model of IR to assess the effects of NHEI on indices of regional and global myocardial contractility and viability when instituted at the time of reperfusion and after a prolonged period of ischemia.

\section{Methods}

\section{Study Rationale/Overview}

The present study used an acute porcine model in which the effects of NHEI on steady-state hemodynamics, myocardial injury, and left ventricular (LV) contractility were assessed after prolonged myocardial IR. Pigs were used because their coronary anatomy and response to myocardial ischemia are similar to those of humans. ${ }^{23}$ The selective NHE inhibitor, cariporide (HOE-642, 4-isopropyl3-methyl-sulfonylbenzoyl-guanidine methanesulphonate, $3 \mathrm{mg} /$ $\mathrm{kg}$ ), was instituted 15 minutes before reperfusion to simulate the common clinical scenario of myocardial infarction in which pharmacologic intervention is administered and coronary reperfusion is reestablished only after a significant duration of ischemia. This dose has previously been shown to confer myocardial protection when administered before the onset of ischemia in experimental myocardial IR. ${ }^{12,15}$ All animals were treated and cared for in accordance with the National Institute of Health Guide for the Care and Use of Laboratory Animals (National Research Council, Washington, 1996).

\section{Experimental Design}

Short-Term instrumentation. Yorkshire pigs $(\mathrm{n}=21,32 \mathrm{~kg})$ were sedated with diazepam (Valium, $200 \mathrm{mg}$ orally) and anesthetized with sufentanyl ( $2 \mu \mathrm{g} / \mathrm{kg}$ intravenously; Baxter Healthcare Corp, Deerfield, Ill) and etomidate $(0.3 \mathrm{mg} / \mathrm{kg}$ intravenously; Bedford Laboratories, Bedford, Ohio). The pigs' lungs were mechanically ventilated and a stable anesthetic plane was achieved with morphine sulfate $(3 \mathrm{mg} / \mathrm{kg}$ per hour intravenously, Elkins-Sinn, Cherry Hill, NJ) and isoflurane $\left(1 \%, 1.5 \mathrm{~L} / \mathrm{min} \mathrm{O}_{2}\right)$. Lactated Ringer's solution $(150 \mathrm{~mL} / \mathrm{h})$ was infused throughout the protocol. An aortic (8F) and venous (7F) line were placed into the carotid artery and internal jugular vein, respectively, to monitor systemic pressures and collect plasma. A multilumen thermodilution catheter (7.5F, Baxter Healthcare Corp, Irvine, Calif) was positioned in the pulmonary artery via the external jugular vein. A median sternotomy was performed and a vessel loop was placed around the inferior vena cava. A precalibrated Micro-Tip transducer (7.5 F, Millar Instruments Inc, Houston, Tex) was advanced into the left ventricle through the apex. Two pairs of piezoelectric crystals ( $2 \mathrm{~mm}$, Sonometrics, London, Ontario, Canada) were positioned against the LV endocardial surface to measure segmental wall motion (Figure 1). Pressure waveforms and crystal signals were digitized for subsequent analysis at a sampling frequency of 100 $\mathrm{Hz}$ (Sonolab, Sonometrics). A snare was placed around the circumflex coronary artery just distal to the first obtuse marginal branch.

Baseline measurements. Steady-state hemodynamic parameters were recorded and LV contractility was determined by using transient inferior vena caval occlusion and sonomicrometry. Specifically, the distance between the crystals placed within each region was recorded at end-diastole and end-systole to compute segmental shortening. These measurements were used to generate region-specific end-systolic pressure-segment distance (dimension, RESPDR) relationships. End-diastolic and end-systolic distances between the remote and area-at-risk region was determined as well to calculate interregional shortening.

Myocardial ischemia. Heparin sodium (150 U/kg intravenously, Pharmacia and Upjohn, Kalamazoo, Mich) and lidocaine $\mathrm{HCl}$ (100 mg intravenously) boluses were administered and the coronary snare was tightened. Regional myocardial ischemia was confirmed by paradoxical LV posterolateral wall motion, tissue blanching, and electrocardiographic changes. Episodes of ventricular tachycardia and fibrillation were terminated with boluses of lidocaine $\mathrm{HCl}$ (50 mg, intravenously) and internal defibrillation, respectively.

Randomization. At 105 minutes of ischemia, the pigs were randomized to IR only $(\mathrm{n}=11,250 \mathrm{~mL}$ of normal saline solution over 15 minutes, intravenously) or NHEI $(n=10$, HOE-642, 3 $\mathrm{mg} / \mathrm{kg}$ in $250 \mathrm{~mL}$ normal saline solution over 15 minutes, intravenously) such that both treatments were completed by 120 minutes of ischemia (Figure 1). 

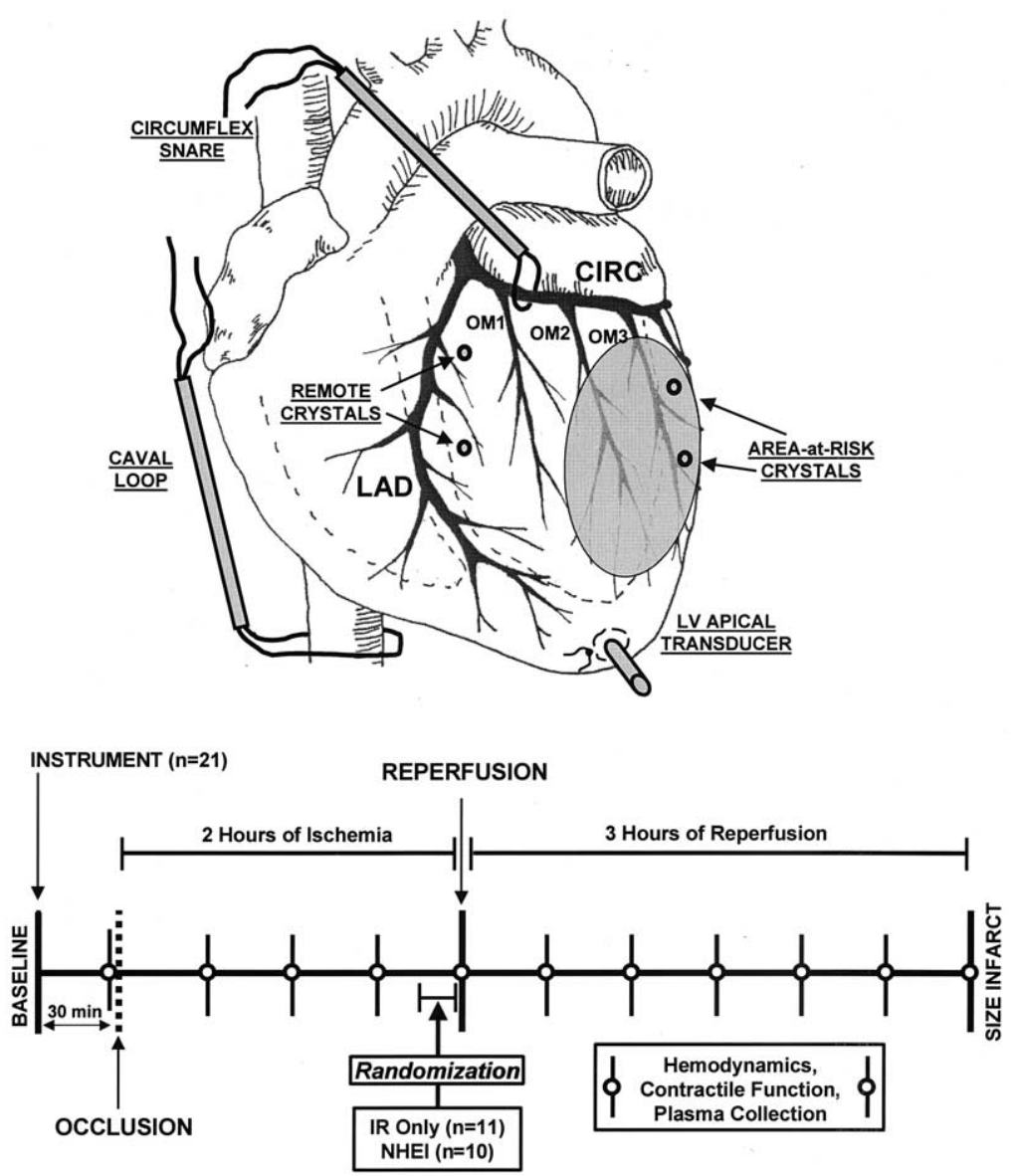

Figure 1. Top, A schematic of the heart after instrumentation. Snares were placed around the inferior vena cava and circumflex coronary artery. Sonomicrometry crystals were positioned along the endocardium of the LV wall within the anterior (remote) and posterolateral (area at risk, shaded) regions. Bottom, Instrumentation was followed by a 30-minute equilibration period. After 105 minutes of ischemia, pigs were randomized to IR only or NHEI (infused for 15 minutes). Reperfusion was initiated 15 minutes later and was continued for 180 minutes. Systemic hemodynamics and indices of LV systolic function was assessed at 30-minute intervals throughout the IR protocol.

Myocardial reperfusion. After 120 minutes of ischemia, the coronary snare was removed and the pigs were reperfused for 180 minutes. Preliminary studies confirmed complete reperfusion of the myocardial area at risk through the use of fluorescent microspheres.

Infarct sizing and plasma profiles. Following euthanasia, the hearts from the pigs were extirpated, and midventricular short-axis sections were obtained to estimate infarct size by triphenyltetrazolium chloride (TTC) staining as previously described. ${ }^{24}$ Infarct size was computed as a percentage of the myocardial area at risk. $^{14,15}$ Plasma troponin-I and creatine kinase MB (CK-MB) levels were assessed by the AxSYM automated microparticle enzyme immunoassay analyzer (Abbott Laboratories, Abbott Park, Ill). Plasma endothelin-1 (ET-1) levels were determined with a high-sensitivity radioimmunoassay as described previously. ${ }^{25}$

Data analysis. The sample size used resulted in a computed power that exceeded 0.80 for indices of LV systolic function. Temporal changes in hemodynamic parameters, systolic function, and plasma enzyme values between the IR-only and NHEI groups were analyzed by a 2-way analysis of variance (ANOVA) followed by mean separation using pairwise Bonferroni corrections. A Pearson-corrected $\chi^{2}$ analysis was used to assess differences between IR groups with respect to a requirement for internal defibrillation and the total number of defibrillation attempts necessary to restore sinus rhythm. Values are presented as the mean and standard error of the mean.

\section{Results}

During the ischemic interval, $18 \%$ and $20 \%$ of the pigs required internal defibrillation in the IR-only and NHEI groups, respectively, with no difference between groups $\left(\chi^{2}\right.$, $P=.92$ ). There was no difference between groups with respect to the total number of defibrillation attempts required to reestablish sinus rhythm $\left(\chi^{2}, P=.96\right)$. No episodes of ventricular fibrillation were observed after coro- 
TABLE 1. Systemic hemodynamics at baseline and following ischemia and reperfusion

\begin{tabular}{|c|c|c|c|c|}
\hline & Baseline & $\begin{array}{l}90 \text { minutes of } \\
\text { ischemia }\end{array}$ & $\begin{array}{l}30 \text { minutes of } \\
\text { reperfusion }\end{array}$ & $\begin{array}{l}180 \text { minutes of } \\
\text { reperfusion }\end{array}$ \\
\hline \multicolumn{5}{|c|}{ Heart rate (beats/min) } \\
\hline IR Only & & & $110 \pm 8(27)$ & $113 \pm 7(24)^{*}$ \\
\hline NHEI & $100 \pm 3(16)$ & $103 \pm 4(21)$ & $105 \pm 5(15)$ & $110 \pm 6(17)$ \\
\hline \multicolumn{5}{|c|}{ Cardiac output (L/min) } \\
\hline IR only & & & $3.0 \pm 0.3(1.0)^{*}$ & $3.8 \pm 0.2(0.6) \dagger, \ddagger$ \\
\hline NHEI & $3.7 \pm 0.2(0.8)$ & $2.9 \pm 0.1(0.7)^{*}$ & $3.0 \pm 0.3(0.9)^{*}$ & $3.1 \pm 0.3(0.9) \S$ \\
\hline \multicolumn{5}{|c|}{ Mean aortic pressure $(\mathrm{mm} \mathrm{Hg})$} \\
\hline IR only & & & $69 \pm 5(17)^{*}, \dagger$ & $64 \pm 2(8)^{*}, \dagger$ \\
\hline NHEI & $88 \pm 3(14)$ & $85 \pm 3(18)$ & $73 \pm 5(16)^{*}, \dagger$ & $69 \pm 3(10)^{*}, \dagger$ \\
\hline \multicolumn{5}{|c|}{ LV peak pressure $(\mathrm{mm} \mathrm{Hg})$} \\
\hline IR only & & & $92 \pm 6(19)^{*}, \dagger$ & $93 \pm 2(6)^{*}, \dagger$ \\
\hline NHEl & $113 \pm 2(11)$ & $109 \pm 3(17)$ & $97 \pm 5(15)^{*}, \dagger$ & $95 \pm 5(15)^{*}, \dagger$ \\
\hline \multicolumn{5}{|c|}{ LV end-diastolic pressure $(\mathrm{mm} \mathrm{Hg})$} \\
\hline IR only & & & $10 \pm 1(3)$ & $10 \pm 1(3)$ \\
\hline NHEI & $10 \pm 1(5)$ & $11 \pm 1(3)$ & $11 \pm 1(3)$ & $11 \pm 1(4)$ \\
\hline \multicolumn{5}{|c|}{$\mathrm{dP} / \mathrm{dt} \max (\mathrm{mm} \mathrm{Hg} / \mathrm{s})$} \\
\hline IR only & & & $1373 \pm 130(432)^{*}$ & $1631 \pm 90(299)$ \\
\hline NHEI & $1654 \pm 78(410)$ & $1520 \pm 51(269)$ & $1625 \pm 156(494)$ & $1598 \pm 183(549)$ \\
\hline \multicolumn{5}{|c|}{$\begin{array}{c}\text { Systemic vascular resistance } \\
\left(\times 10^{-3} \text { dyne } \cdot \mathrm{s} \cdot \mathrm{cm}^{-5}\right)\end{array}$} \\
\hline IR only & & & $2.0 \pm 0.2(0.7) \dagger$ & $1.4 \pm 0.1(0.3)^{*}, \dagger, \ddagger$ \\
\hline NHEI & $2.0 \pm 0.1(0.5)$ & $2.5 \pm 0.2(0.8)^{*}$ & $2.1 \pm 0.2(0.6)$ & $1.9 \pm 0.1(0.4) \dagger, \S$ \\
\hline \multicolumn{5}{|l|}{ Sample size } \\
\hline IR only & & & 11 & 11 \\
\hline NHEI & 21 & 21 & 10 & 9 \\
\hline
\end{tabular}

Values presented as mean \pm SEM (standard deviation). NHEl (cariporide, $3 \mathrm{mg} / \mathrm{kg}$ ) infused over 15 minutes before reperfusion.

${ }^{*} P<.05$ versus baseline.

$\dagger P<.05$ versus 90 -minute postischemia.

$\ddagger P<.05$ versus 30 -minute postreperfusion.

$\S P<.05$ versus time-matched IR only values).

nary snare release. One pig in the NHEI group died of hemodynamic collapse at 150 minutes of reperfusion, resulting in the sample sizes demonstrated in Table 1. Importantly, all data collected from this animal, including infarct size, were included and analyzed in the investigation.

\section{Steady-State Hemodynamics}

Steady-state hemodynamic measurements are summarized in Table 1. Because the pigs were treated in identical fashion for the first 90 minutes of ischemia and analysis of variance revealed no difference between groups before randomization, the measurements obtained from these initial time points were pooled. At 180 minutes of reperfusion, heart rate was increased, and mean arterial pressure and systemic vascular resistance were decreased in the IR-only group compared with baseline. Compared with IR-only values, cardiac output decreased and systemic vascular resistance increased in the NHEI group.

\section{Intraregional Function}

By 30 minutes of ischemia, regional shortening and the RESPDR were decreased from baseline values within the area at risk in both IR groups (Figure 2). However, by 30 minutes of reperfusion, regional shortening was increased in the NHEI group compared with IR-only values. Moreover, the RESPDR was higher in the NHEI group within 15 minutes of NHEI infusion compared with the IR-only group. Within the remote region, there were no changes in regional shortening or the RESPDR in either IR group.

\section{Interregional Function}

Regional shortening was determined between the remote and area-at-risk region as an assessment of global LV systolic function (Figure 3). Interregional shortening was reduced at 90 minutes of ischemia in both IR groups. However, by 120 minutes of ischemia, and throughout the reperfusion interval, interregional shortening was increased in the NHEI group compared with IR-only values.

\section{Infarct Sizes and Plasma Profiles}

Infarct size, computed as a percentage of the area at risk, was $39 \% \pm 6 \%$, and there was no difference between IR groups $(P=.26)$. The plasma levels of the myocyte-specific enzymes, cardiac troponin-I and CK-MB, and the potent 

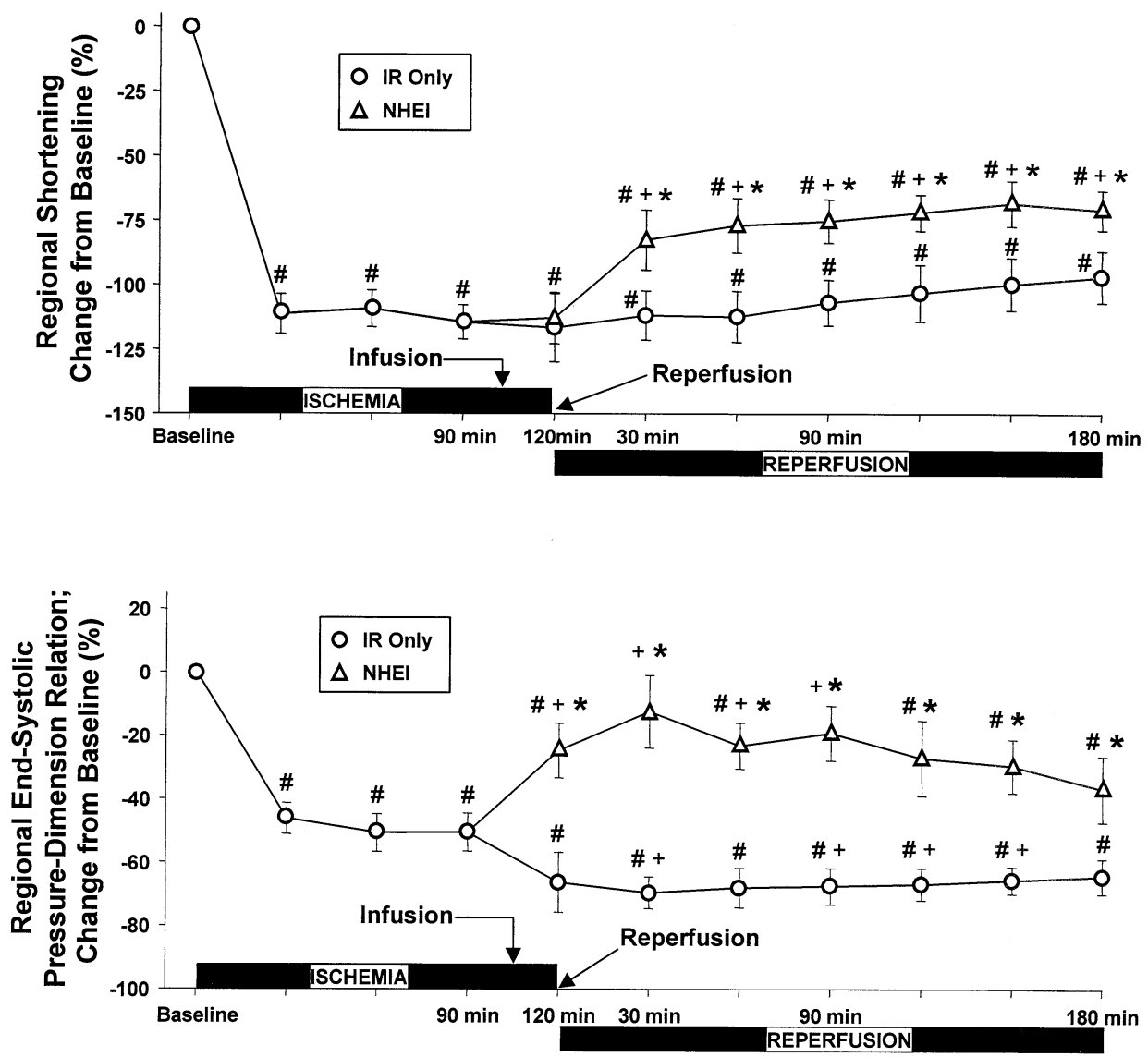

Figure 2. Regional shortening (top) and the regional end-systolic pressure-dimension relation (RESPDR; bottom) obtained from the area at risk are presented as changes from baseline values. At $\mathbf{9 0}$ minutes of ischemia, regional shortening and the RESPDR were decreased in both IR groups. By 30 minutes of reperfusion, regional shortening was increased in the NHEI group compared with IR-only values. With respect to the RESPDR, functional improvements were observed in the NHEl group within 15 minutes of NHEI infusion compared to the IR-only group. These differences persisted throughout the reperfusion period. ${ }^{\#} \boldsymbol{P}<.05$ versus baseline; ${ }^{+} \boldsymbol{P}<.05$ versus 90 minutes of ischemia; ${ }^{*} P<.05$ versus IR only.

vasoconstrictor peptide ET-1 were serially measured during the IR protocol and are summarized in Figure 4. Robust increases in troponin-I and CK-MB levels were observed in both IR groups immediately after removal of the coronary snare. ET-1 levels similarly increased in both IR groups during the ischemic interval. There were no differences in enzyme plasma levels between the IR groups.

\section{Discussion}

Past animal studies suggest that NHE activation contributes to IR-mediated myocardial injury and dysfunction. ${ }^{12-18}$ However, these studies did not examine the effects of NHEI on myocardial injury and function when instituted at reperfusion following prolonged ischemia. An evaluation of this approach may be more clinically relevant as suggested by recent investigations in patients with prolonged myocardial IR. ${ }^{19-22}$ Accordingly, the goal of the present study was to examine the role of NHEI in a setting of prolonged myocardial IR using an experimental design and animal model that more closely recapitulate the clinical context. The unique findings of the present investigation are 2-fold. First, when instituted at reperfusion, NHEI was associated with significant and sustained improvements in regional and global LV systolic function. Second, these improvements occurred independent of the degree of myocardial injury sustained during IR as assessed by absolute infarct size and myocyte-specific enzyme release.

\section{Contractility After IR: Effects of NHEI}

Previous experimental investigations of myocardial IR demonstrated that NHEI improved viability and indices of LV systolic function when administered before, or shortly after, the onset of ischemia. ${ }^{12-18}$ However, the majority of these studies used short durations of ischemia and administered 

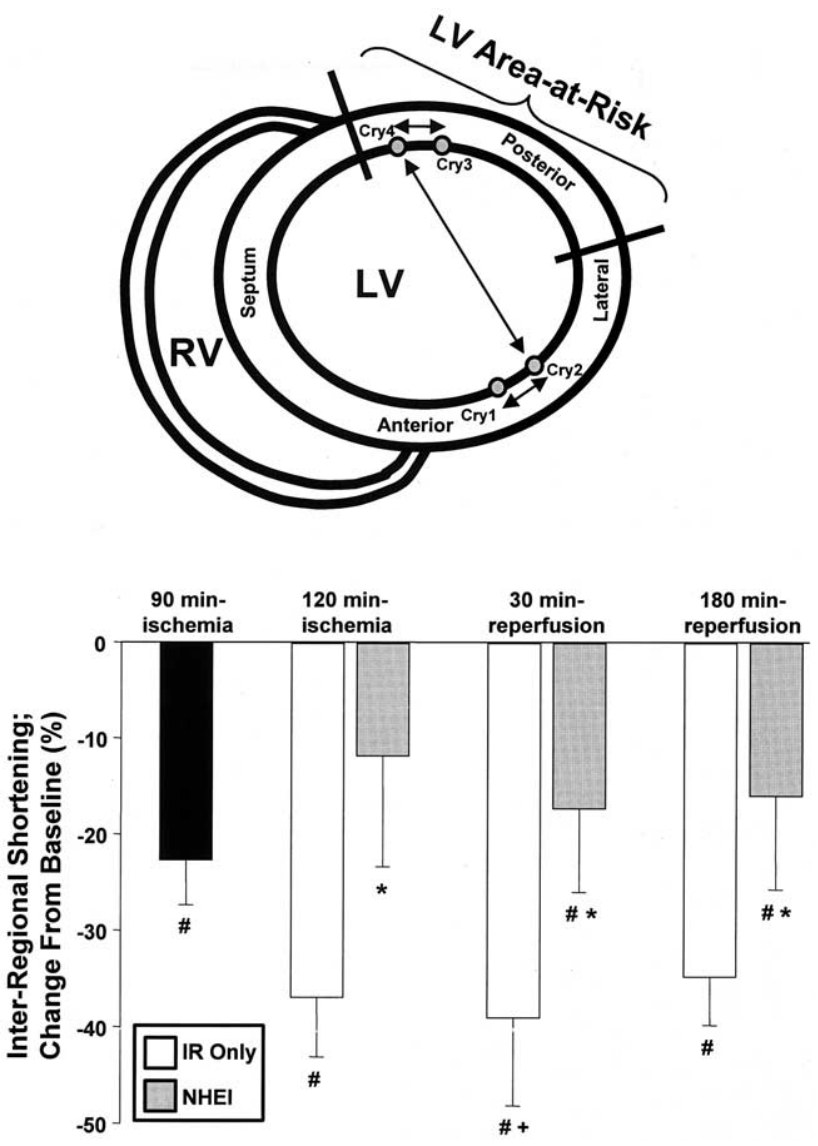

Figure 3. Top, A schematic depicting the instrumented left ventricle as observed from a view perpendicular to the LV long-axis. Sonomicrometry crystals are shown in their approximate endocardial positions in the remote and area-at-risk regions. Regional shortening was determined between these regions. Because the distance between the remote and area-at-risk regions approximated the diameter of the left ventricle, this assessment of interregional shortening represented an estimate of global LV systolic function. Bottom, Interregional shortening presented as a change from baseline values. Shortening was reduced at $\mathbf{9 0}$ minutes of ischemia in both IR groups. By 120 minutes of ischemia and throughout reperfusion, interregional shortening was increased in the NHEI group compared with IR-only values. ${ }^{\#} \boldsymbol{P}<.05$ versus baseline; ${ }^{+} \boldsymbol{P}<.05$ versus 90 minutes of ischemia; ${ }^{*} \boldsymbol{P}<.05$ versus IR only. $L V$, Left ventricle (ventricular); $R V$, right ventricle.

NHEI at the onset of coronary occlusion, thus limiting extrapolation to the clinical context. Moreover, load-independent indices of LV contractility, such as pressure-dimension relationships, were not examined. Therefore, whether and to what degree NHEI influenced LV contractile performance independent of infarct size and myocyte-specific enzyme release when administered after a prolonged period of ischemia and at the time of reperfusion remain unknown. The present investigation built on these past experimental IR studies and directly addressed this issue in the following ways. First, an animal model and experimental paradigm that closely recapitulated the common clinical context of myocardial IR was used. Second, changes in LV pump performance after IR were evaluated by the use of indices of LV contractility such as end-systolic pressure-dimension relationships, a load independent index of regional systolic performance. The unique results of the present study demonstrated that NHEI improved indices of regional and global LV contractility after IR in the absence of significant effects on infarct size and myocyte-specific enzyme release. Importantly, although the results of this study mirror those of recent clinical trials with respect to myocardial injury, ${ }^{21,22}$ the observed improvement in LV systolic function contrasts corresponding results from the same clinical investigations. This disconnect is likely attributable to differences in the experimental designs. Specifically, the present investigation directly interrogated the myocardium during the acute IR period while past clinical trials used less direct means to assess LV function weeks after the initial myocardial insult. ${ }^{19,20}$

\section{Myocardial Injury After IR: Effects of NHEI}

In the present study, NHEI failed to significantly attenuate infarct size when instituted at the time of coronary reperfusion as assessed by postmortem histochemical staining. ${ }^{24}$ Past experimental studies of IR demonstrated similar failures of myocardial protection when NHEI was administered before reperfusion but after significantly shorter periods of ischemia. ${ }^{13-15}$ However, the serial evaluation of plasma levels of myocyte-specific enzymes was not performed in these past studies. Accordingly, to more closely evaluate the degree of myocardial injury inflicted by the IR event and the potential role of NHEI in the manifestation of this process, the present study used temporal plasma profiling of the myocyte-specific enzymes troponin-I and CK-MB. Robust increases in the levels of these enzymes were observed in both IR groups after snare release. There was no significant difference in the temporal release of these enzymes between the IR groups during the reperfusion period. Nevertheless, despite equivalent degrees of release between groups, indices of regional and global LV contractility were improved with NHEI at the time of reperfusion, suggesting that the observed gains in systolic function occurred independent of myocardial viability.

\section{Mechanism for Improved LV Function After IR}

Ischemia induces perturbations in acid-base homeostasis within myocytes that may ultimately translate into deteriorations in LV contractile performance. The NHE, through a reversible ion-exchange mechanism, removes intracellular $\mathrm{H}^{+}$that accumulates during periods of ischemia in exchange for extracellular $\mathrm{Na}^{+} .8,21,26$ Importantly, this compensatory ionic mechanism is coupled to an additional and 

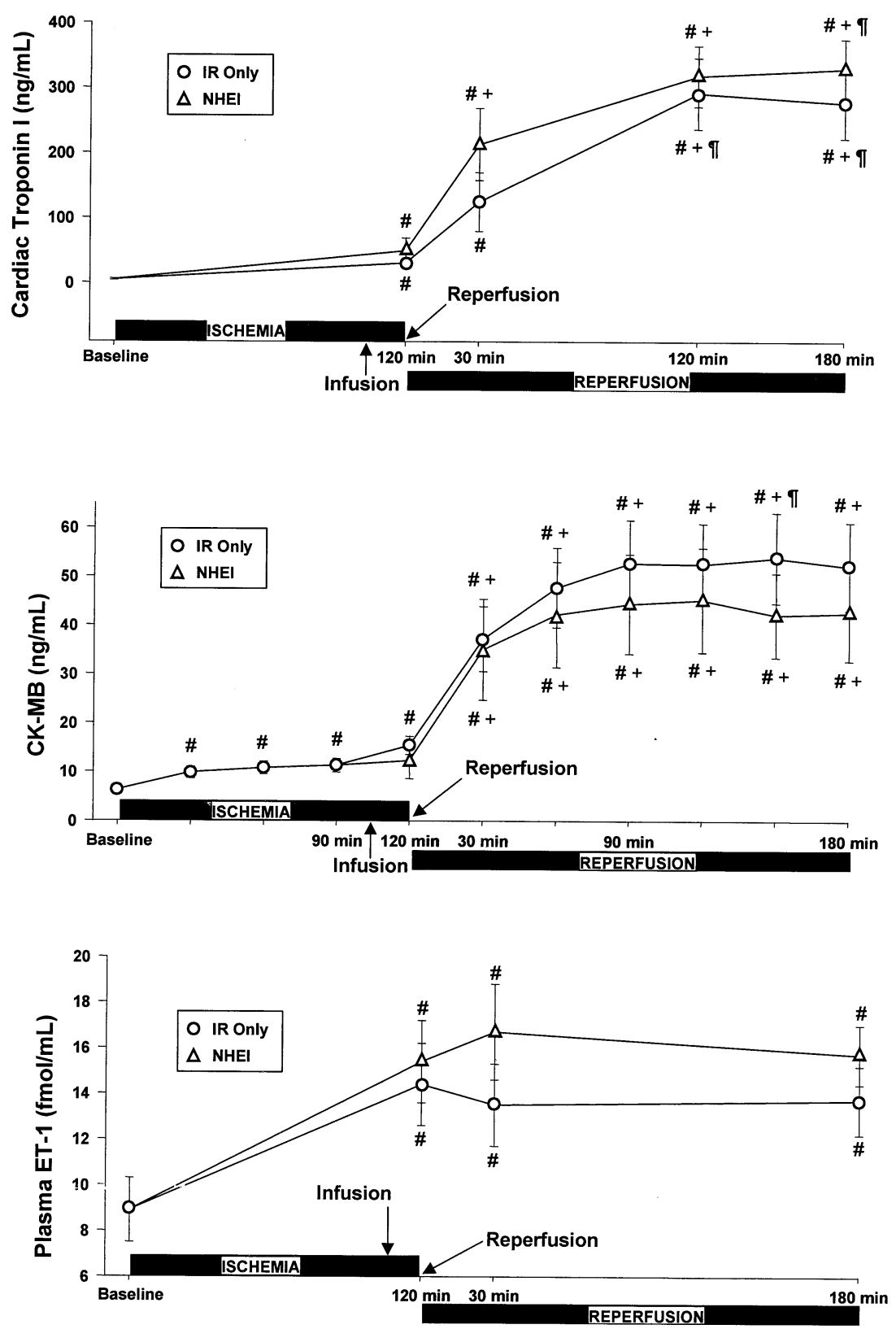

Figure 4. Plasma levels of troponin-I (top), creatine kinase MB (CK-MB, middle), and endothelin-1 (ET-1, bottom) were assessed at baseline and during the IR protocol. Marked increases in plasma values of troponin-I and CK-MB were apparent immediately after myocardial reperfusion, suggesting that the occluded arteries were immediately patent after snare removal. There were no differences in troponin-I, CK-MB, or ET-1 profiles between the IR groups, suggesting that equivalent and significant degrees of myocardial ischemia and injury were produced. ${ }^{\#}<.05$ versus baseline; ${ }^{+} \boldsymbol{P}<.05$ versus 90 minutes of ischemia; $\boldsymbol{P}<.05$ versus 30 minutes of reperfusion.

distinct ion exchanger that responds to increases in intracellular $\mathrm{Na}^{+}$by facilitating additional ion exchange with extracellular $\mathrm{Ca}^{2+} \cdot{ }^{11,19,27}$ Thus, the end result of prolonged myocardial ischemia is detrimental accumulation of intracellular $\mathrm{Ca}^{2+} .{ }^{10}$ Increased NHE activity during prolonged IR may exacerbate LV contractile performance by contrib- uting to abnormalities in $\mathrm{Ca}^{2+}$ sensitivity and homeostasis. ${ }^{27}$ The present study used NHEI at the time of reperfusion to interrupt this ion-exchange process and abrogate the effects of excessive $\mathrm{Ca}^{2+}$ accumulation on LV contractility. Despite a prolonged period of ischemia, NHEI was associated with improved regional and global myocardial contrac- 
tility when instituted at the time of reperfusion compared with peak-ischemic and IR-only values. This beneficial effect was likely achieved through attenuations in myocyte calcium accumulation during the early reperfusion period that translated into improved $\mathrm{Ca}^{2+}$ sensitivity and homeostasis. However, this issue remains speculative and warrants further investigation.

\section{Steady-State Hemodynamics/Neurohormonal Activation}

NHEI administered at reperfusion did not appear to adversely influence steady-state hemodynamics. However, NHEI was associated with increased systemic vascular resistance compared with IR-only values, which may have contributed to the slight, yet significant, decrease in cardiac output associated with the NHEI group. The change in systemic vascular resistance was likely due to the systemic effects of NHEI on vascular smooth muscle. These systemic effects not withstanding, significant improvements in regional and global LV contractility were observed with NHEI administration at reperfusion. Importantly, the beneficial effects conferred by NHEI were not the result of chronotropic manipulation during the reperfusion interval.

A common physiologic response to myocardial IR is activation of the neurohormonal system. For example, increased synthesis and/or release of the potent bioactive peptide endothelin (ET) have been observed in settings of myocardial IR. ${ }^{28}$ Moreover, elevated ET levels have been suggested to exacerbate LV systolic dysfunction in numerous cardiovascular disease states, ${ }^{29}$ and ET receptor activation has been shown to result in increased activity of the NHE. ${ }^{30}$ The present study demonstrated that ET levels were elevated at the time of myocardial reperfusion. Although NHEI had no effect on the upstream elaboration of this peptide, it was associated with improvements in LV systolic function in the face of increased ET levels.

\section{Study Limitations and Clinical Implications}

The present study used NHEI in a short-term model of myocardial IR. Therefore, whether and to what degree NHEI influences LV contractility beyond the acute reperfusion period remains to be determined. Moreover, repetitive NHEI dosing during the early reperfusion interval may have further enhanced the beneficial effects observed in LV contractile function. The dose of cariporide infused in this study was similar to that previously used in preclinical investigations $\mathrm{s}^{12,13,15}$ but exceeded that used in recent clinical trials. ${ }^{19,20,22}$ Finally, direct intracoronary infusion of cariporide was not assessed. Nevertheless, the results of the present investigation suggest that an ionic mechanism for myocardial dysfunction after prolonged IR may be increased NHE activity. Moreover, these results suggest that the administration of NHEI at the time of myocardial reperfusion does not preclude the realization of beneficial effects on LV systolic function. Rather, NHEI may serve as a useful adjunct in settings of prolonged IR intervals in which complex percutaneous coronary interventions and coronary artery bypass grafting procedures are performed. These results extend the potential benefits of NHEI institution in the IR setting beyond that of myocardial salvage alone. Thus, modulation of the NHE may hold therapeutic potential in the setting of IR.

\section{References}

1. ISIS-2 (Second International Study of Infarct Survival) Collaborative Group. Randomised trial of intravenous streptokinase, oral aspirin, both, or neither among 17,187 cases of suspected acute myocardial infarction: ISIS-2. Lancet. 1988;2:349-60.

2. Weaver WD, Simes RJ, Betriu A, et al. Comparison of primary coronary angioplasty and intravenous thrombolytic therapy for acute myocardial infarction: a quantitative review. JAMA. 1997;278:2093-8.

3. Braunwald E, Kloner RA. Myocardial reperfusion: a double-edged sword? J Clin Invest. 1985;76:1713-9.

4. Wang QD, Pernow J, Sjoquist PO, Ryden L. Pharmacological possibilities for protection against myocardial reperfusion injury. Cardiovasc Res. 2002;55:25-37.

5. Fliss H, Gattinger D. Apoptosis in ischemic and reperfused rat myocardium. Circ Res. 1996;79:949-56.

6. Zhao ZQ, Nakamura M, Wang NP, et al. Reperfusion induces myocardial apoptotic cell death. Cardiovasc Res. 2000;45:651-60.

7. Karmazyn M, Sostaric JV, Gan XT. The myocardial $\mathrm{Na}^{+} / \mathrm{H}^{+}$exchanger: a potential therapeutic target for the prevention of myocardial ischaemic and reperfusion injury and attenuation of postinfarction heart failure. Drugs. 2001;61:375-89.

8. Lazdunski M, Frelin C, Vigne P. The sodium/hydrogen exchange system in cardiac cells: its biochemical and pharmacological properties and its role in regulating internal concentrations of sodium and internal pH. J Mol Cell Cardiol. 1985;17:1029-42.

9. Scholz W, Albus U, Lang HJ, et al. Hoe 694, a new $\mathrm{Na}^{+} / \mathrm{H}^{+}$exchange inhibitor and its effects in cardiac ischaemia. Br J Pharmacol. 1993; 109:562-8.

10. Tani M, Neely JR. $\mathrm{Na}^{+}$accumulation increases $\mathrm{Ca}^{2+}$ overload and impairs function in anoxic rat heart. J Mol Cell Cardiol. 1990;22:57-72.

11. Inserte J, Garcia-Dorado D, Ruiz-Meana M, et al. Effect of inhibition of $\mathrm{Na}^{(+)} / \mathrm{Ca}^{(2+)}$ exchanger at the time of myocardial reperfusion on hypercontracture and cell death. Cardiovasc Res. 2002;55:739-48.

12. Portman MA, Panos AL, Xiao Y, Anderson DL, Ning X. HOE-642 (cariporide) alters $\mathrm{pH}_{\mathrm{i}}$ and diastolic function after ischemia during reperfusion in pig hearts in situ. Am J Physiol Heart Circ Physiol. 2001;280:H830-4.

13. Garcia-Dorado D, Gonzalez MA, Barrabes JA, et al. Prevention of ischemic rigor contracture during coronary occlusion by inhibition of $\mathrm{Na}^{+}-\mathrm{H}^{+}$exchange. Cardiovasc Res. 1997;35:80-9.

14. Klein HH, Pich S, Bohle RM, Lindert-Heimberg S, Nebendahl K. $\mathrm{Na}^{+} / \mathrm{H}^{+}$exchange inhibitor cariporide attenuates cell injury predominantly during ischemia and not at onset of reperfusion in porcine hearts with low residual blood flow. Circulation. 2000;102:1977-82.

15. Klein HH, Pich S, Bohle RM, Wollenweber J, Nebendahl K. Myocardial protection by $\mathrm{Na}^{+}-\mathrm{H}^{+}$exchange inhibition in ischemic, reperfused porcine hearts. Circulation. 1995;92:912-7.

16. Rohmann S, Weygandt H, Minck KO. Preischaemic as well as postischaemic application of a Na${ }^{+} / \mathrm{H}^{+}$exchange inhibitor reduces infarct size in pigs. Cardiovasc Res. 1995;30:945-51.

17. Gumina RJ, Mizumura T, Beier N, Schelling P, Schultz JJ, Gross GJ. A new sodium/hydrogen exchange inhibitor, EMD 85131, limits infarct size in dogs when administered before of after coronary artery occlusion. J Pharmacol Exp Ther. 1998;286:175-83.

18. Gumina RJ, Buerger E, Eickmeier C, Moore J, Daemmgen J, Gross GJ. Inhibition of the $\mathrm{Na}^{(+)} / \mathrm{H}^{(+)}$exchanger confers greater cardioprotection against 90 minutes of myocardial ischemia than ischemic preconditioning in dogs. Circulation. 1999;100:2519-26. 
19. Buerke M, Rupprecht HJ, vom Dahl J, et al. Sodium-hydrogen exchange inhibition: novel strategy to prevent myocardial injury following ischemia and reperfusion. Am J Cardiol. 1999;83:19G-22.

20. Rupprecht HJ, vom Dahl J, Terres W, et al. Cardioprotective effects of the $\mathrm{Na}^{(+)} / \mathrm{H}^{(+)}$exchange inhibitor cariporide in patients with acute anterior myocardial infarction undergoing direct PTCA. Circulation. 2000;101:2902-8.

21. Zeymer U, Suryapranata H, Monassier JP, et al. The $\mathrm{Na}^{(+)} / \mathrm{H}^{(+)}$ exchange inhibitor eniporide as an adjunct to early reperfusion therapy for acute myocardial infarction. Results of the evaluation of the safety and cardioprotective effects of eniporide in acute myocardial infarction (ESCAMI) trial. J Am Coll Cardiol. 2001;38:1644-50.

22. Theroux P, Chaitman BR, Danchin N, et al. Inhibition of the sodiumhydrogen exchanger with cariporide to prevent myocardial infarction in high-risk ischemic situations. Main results of the GUARDIAN trial. Guard during ischemia against necrosis (GUARDIAN) Investigators. Circulation. 2000;102:3032-8.

23. Weaver ME, Pantely GA, Bristow JD, Ladley HD. A quantitative study of the anatomy and distribution of coronary arteries in swine in comparison with other animals and man. Cardiovasc Res. 1986;20:907-17.
24. Ytrehus K, Liu Y, Tsuchida A, et al. Rat and rabbit heart infarction: effects of anesthesia, perfusate, risk zone, and method of infarct sizing. Am J Physiol. 1994;267:H2383-90.

25. Ergul A, Walker CA, Goldberg A, et al. ET-1 in the myocardial interstitium: relation to myocyte ECE activity and expression. Am J Physiol Heart Circ Physiol. 2000;278:H2050-6.

26. Frelin $\mathrm{C}$, Vigne $\mathrm{P}$, Lazdunski $\mathrm{M}$. The role of the $\mathrm{Na}^{+} / \mathrm{H}^{+}$exchange system in cardiac cells in relation to the control of the internal $\mathrm{Na}^{+}$ concentration. A molecular basis for the antagonistic effect of ouabain and amiloride on the heart. J Biol Chem. 1984;259:8880-5.

27. Avkiran M. Rational basis for use of sodium-hydrogen exchange inhibitors in myocardial ischemia. Am J Cardiol. 1999;83:10G-7.

28. Pearl JM, Nelson DP, Wagner CJ, Lombardi JP, Duffy JY. Endothelin receptor blockade reduces ventricular dysfunction and injury after reoxygenation. Ann Thorac Surg. 2001;72:565-70.

29. Luscher TF, Barton M. Endothelins and endothelin receptor antagonists: therapeutic considerations for a novel class of cardiovascular drugs. Circulation. 2000;102:2434-40.

30. Fliegel L. Regulation of myocardial $\mathrm{Na}^{+} / \mathrm{H}^{+}$exchanger activity. Basic Res Cardiol. 2001;96:301-5.

\section{Targeted}

The Journal of Thoracic and Cardiovascular Surgery gives you two tables of contents.

The condensed table of contents tells you at a glance what topics and authors are presented each month. The expanded table of contents gives you a brief abstract of each article. You select only those articles of most interest to you for further reading. 